Provided for non-commercial research and education use. Not for reproduction, distribution or commercial use.

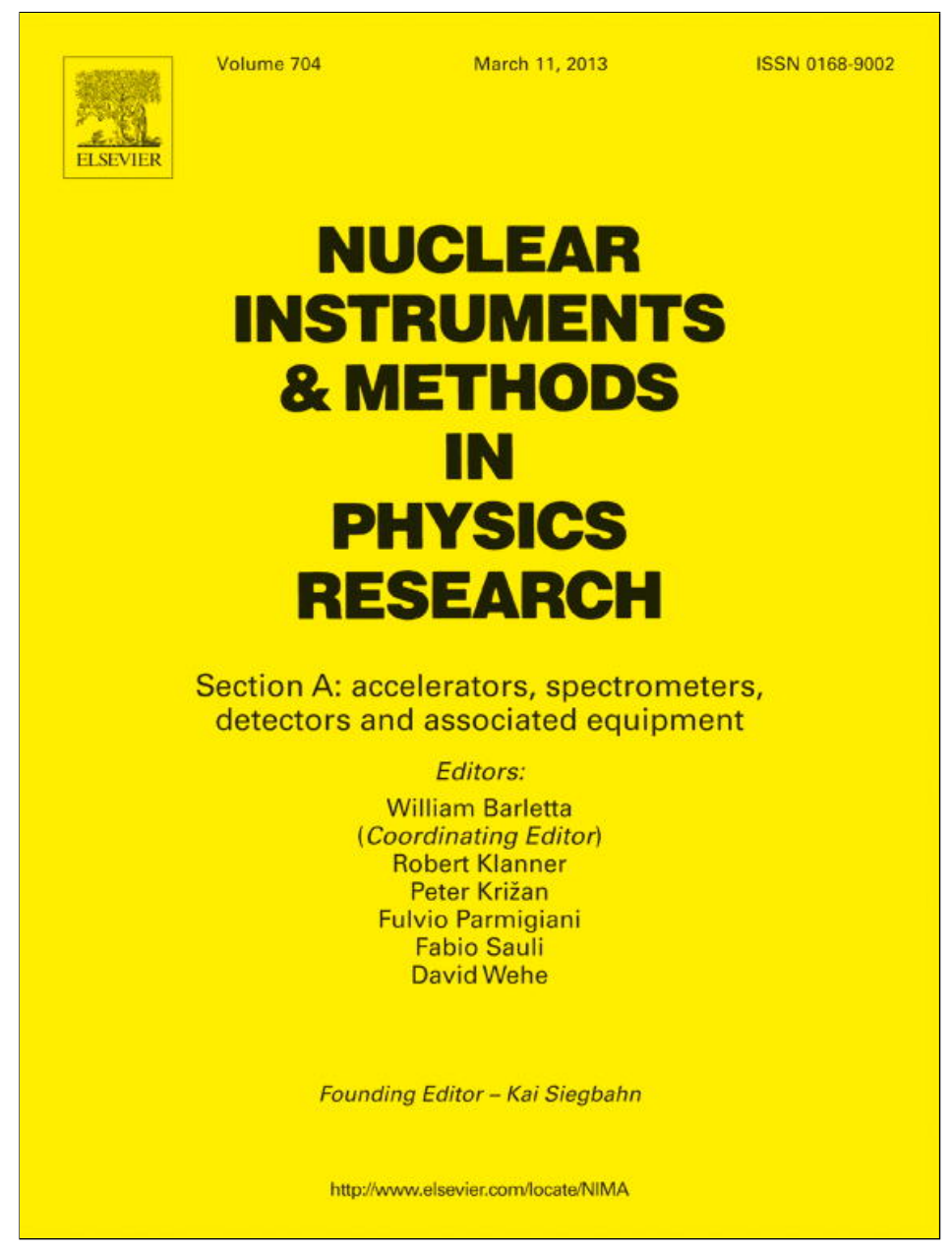

This article appeared in a journal published by Elsevier. The attached copy is furnished to the author for internal non-commercial research and education use, including for instruction at the authors institution and sharing with colleagues.

Other uses, including reproduction and distribution, or selling or licensing copies, or posting to personal, institutional or third party websites are prohibited.

In most cases authors are permitted to post their version of the article (e.g. in Word or Tex form) to their personal website or institutional repository. Authors requiring further information regarding Elsevier's archiving and manuscript policies are encouraged to visit:

http://www.elsevier.com/copyright 


\title{
From neutron Compton profiles to momentum distribution: Assessment of direct numerical determination
}

\author{
R. Senesi ${ }^{\mathrm{a}, \mathrm{b}, \mathrm{c}, *}, \mathrm{D}$. Flammini ${ }^{\mathrm{a}, \mathrm{b}}, \mathrm{G}$. Romanelli ${ }^{\mathrm{a}, \mathrm{b}}, \mathrm{C}$. Andreani ${ }^{\mathrm{a}, \mathrm{b}, \mathrm{c}}$ \\ a Università degli Studi di Roma "Tor Vergata”, Dipartimento di Fisica, Via della Ricerca Scientifica 1, 00133 Roma, Italy

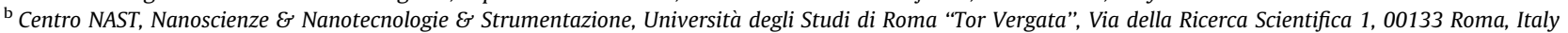 \\ ${ }^{\mathrm{c}}$ CNR-IPCF, Italy
}

\section{A R T I C L E I N F O}

\section{Article history:}

Received 3 October 2012

Received in revised form

5 November 2012

Accepted 4 December 2012

Available online 10 December 2012

Keywords:

Inelastic neutron scattering

Momentum distributions

Neutron Compton scattering

\begin{abstract}
A B S T R A C T
Inelastic neutron scattering at high momentum transfers, in the neutron Compton scattering regime, provides an access to the neutron Compton profiles, the analogous of Compton profiles in X-ray scattering. The line shape analysis of the neutron Compton profiles is usually carried out making use of multiparametric nonlinear fitting, garnering detailed information about the momentum distribution of the target atoms. This paper presents the proposal to directly determine numerically the momentum distribution from the profiles, thus eliminating the possible instabilities present in multiparametric fitting. A comparison with Monte Carlo simulations and with previous measurements on polycrystalline ice provides quantitative assessments of the proposed method.
\end{abstract}

(c) 2012 Elsevier B.V. All rights reserved.

\section{Introduction}

Since the early development of X-ray Compton scattering, the realisation that the target atom in the systems necessarily contains bound electrons, which cannot be stationary, led to the interpretation that Compton scattered beams were Doppler broadened due to the motion of the target electrons [1]. Du Mond used the novel Fermi-Dirac distribution function to predict the line shape for beryllium samples [2]. His results possibly constitute the earliest direct evidence for the validity of the Fermi-Dirac statistics for the electron gas. Thus, for target electrons possessing a probability density distribution $n(\mathbf{p})$, the Compton profile, $J\left(p_{z}\right)$, where $z$ is the direction of the scattering vector, is the projection of $n(\mathbf{p})$ along the scattering vector:

$J\left(p_{z}\right)=\int_{p_{x}} \int_{p_{y}} n\left(p_{x}, p_{y}, p_{z}\right) d p_{x} d p_{y}$

In isotropic systems $n(\mathbf{p})$ depends only on the magnitude of $\mathbf{p}$, and it can be shown that [3]

$J\left(p_{z}\right)=2 \pi \int_{|y|}^{\infty} p n(p) d p$

\footnotetext{
* Corresponding author at: Università degli Studi di Roma "Tor Vergata", Dipartimento di Fisica, Via della Ricerca Scientifica 1, 00133 Roma, Italy. Tel.: +3906 72594549; fax: +39062023507.

E-mail address: roberto.senesi@uniroma2.it (R. Senesi).
}

and hence that

$n(p)=-\left.\frac{1}{2 \pi p_{z}} \frac{d J\left(p_{z}\right)}{d p_{z}}\right|_{p z=p}$

the latter expression for the momentum distribution in terms of the derivative of the Compton profile is originally due to $\mathrm{Du}$ Mond [2]. Inelastic neutron scattering at high wave vector and eV energy transfers [4] provides access to the Deep Inelastic Neutron Scattering (DINS), or neutron Compton Scattering (NCS), regime $[5,6]$. Here the momentum distribution of atomic nuclei is probed, with the scattering law being described within the same framework (impulse approximation) as X-Ray Compton scattering [7]: the energy, $\hbar \omega$, and momentum, $\hbar \mathbf{q}$, transfers are related by

$\hbar \omega=\frac{\left(\mathbf{p}_{\mathbf{n}}-\mathbf{p}_{\mathbf{n}}^{\prime}\right)^{2}}{2 M}+\frac{\left(\mathbf{p}_{\mathbf{n}}-\mathbf{p}_{\mathbf{n}}^{\prime}\right) \cdot \mathbf{p}}{M}$

where $M$ and $\mathbf{p}$ are the mass and the momentum of struck particle before collision, whereas $\mathbf{p}_{\mathbf{n}}$ and $\mathbf{p}_{\mathbf{n}}^{\prime}$ are the momenta of the incident and scattered neutron (i.e. $\mathbf{p}_{\mathbf{n}}-\mathbf{p}_{\mathbf{n}}^{\prime}=\hbar \mathbf{q}$ ), respectively. Rearranging in terms of the recoil energy of the struck nucleus:

$\hbar \omega_{r}=\frac{\hbar^{2} q^{2}}{2 M}$

It is possible to define a scaling variable, first introduced by West [8], that couples the two dynamic variables $\omega$ and $q$ and represents the component of the atomic momentum along the scattering vector (i.e. $p_{z}$ in the X-Ray formalism above):

$y=\frac{M}{\hbar^{2} q}\left(\hbar \omega-\hbar \omega_{r}\right)=\hat{\mathbf{q}} \cdot \mathbf{p}$ 
The momentum distribution-broadened recoil profile is therefore referred to as Neutron Compton Profile [6]. In real experimental situations the wave vector transfer is finite: for example, on the Vesuvio spectrometer at ISIS, $q \geq 25 \AA^{-1}$. Therefore deviations from the impulsive approximations occur, generally referred to as Final State Effects (FSE) [3]. FSE are due to the localisation of the struck atom in its final state, by surrounding atoms. The overall effect is a broadening of the observed $J(y)$, which is similar to that introduced by an instrumental resolution effect. When FSE are present, the Neutron Compton Profile becomes $q$ dependent, i.e. $J(y)$ needs to be replaced by $J(y, q)$ for isotropic systems. Representing the FSE as a series in powers of $1 / q$, one finds for the isotropic case according to Sears [3]

$J(y, q)=J(y)-\frac{A_{3}}{q} \frac{d^{3}}{d y^{3}} J(y)+\frac{A_{4}}{q^{2}} \frac{d^{4}}{d y^{4}} J(y)+\cdots$

where the coefficients $A_{n}$ are related to the interatomic potential [3]. The measurements on a variety of systems show that FSE are effectively well described by a power series of $q$, and are a few percent of intensity as compared to the intrinsic neutron Compton profile [9]. The second broadening term of the neutron Compton profile is the instrumental resolution, $R_{l}(y, q)$, which depends on $y, q$ at a particular scattering angle $l$. For proton momentum distribution studies on VESUVIO, the FWHM of the experimental Compton profiles is a factor $\geq 6$ larger than the FWHM of the resolution line shape. These two broadening terms are therefore a minor contribution to the observed profiles, with the resolution width of increasing magnitude as the recoiling mass increases $[9,10]$. Thus, the profile measured at a particular scattering angle $l$ is

$F_{l}(y, q)=\left[J(y)+\Delta J_{l}(y, q)\right] \otimes R_{l}(y, q)$

$\Delta J_{l}(y, q)$ are the $q$-dependent deviations from the impulse approximation. This expression is adopted to represent DINS spectra using both direct-geometry chopper spectrometers [11-17], as well as for inverse geometry instruments such as VESUVIO $[5,9,18,19]$. The DINS data analysis is generally carried out using parametric methods, such as nonlinear fitting of the $F_{l}(y, q)$, using model functions for $J(y)$. The most relevant model functions used are the Gauss-Hermite expansion, or the multivariate Gaussian; the former representing a model-independent method and the latter representing anisotropic harmonic motions, such as a light particle confined in harmonic potentials, e.g. the proton in ice Ih $[5,20]$. The nonlinear fitting is applied to the set of fixed angle experimental Compton profiles, in order to minimise the following chi-square:

$\chi^{2}=\Sigma_{l} \Sigma_{i} \frac{\left(F_{l}^{t h}\left(y_{i}, q_{i}\right)-F_{l}\left(y_{i}, q_{i}\right)\right)^{2}}{\epsilon_{l, i}^{2}}$

where the model function is $F_{l}^{t h}\left(y_{i}, q_{i}\right)=\left[J\left(y_{i}\right)+\Delta J_{l}\left(y_{i}, q_{i}\right)\right] \otimes R_{l}\left(y_{i}, q_{i}\right)$. Here the index $l$ represents the detector index, the index $i$ represents the $y$ value at the $i$-th bin, and $\epsilon_{l, i}^{2}$ is the experimental uncertainty for each data point. The fit generally includes a large number of parameters: those relative to the intrinsic profile $J(y)$, such as the standard deviation, non-Gaussian terms, those describing the final state effect terms $\left(A_{n}\right)$, as well as centroids shifts and normalisation constants for each of the $F_{l}(y, q)$, for a total number of parameters approaching 100 .

In order to complement and provide a more direct access to the full line shape of the underlying momentum distribution, in recent years, non-parametric methods have been proposed and applied for the analysis of the neutron Compton profiles: (1) a direct extraction of the variance of the momentum distribution through numerical integration of the detector-averaged Compton profiles, $\bar{F}(y)=\sum_{l=1}^{n} F_{l}(y, q)(1 / n)$, where $n$ is the total number of detectors [17,20-22]; (2) determination of the mean force, $f(x)=(-\log n(x))^{\prime}-M x / \beta \hbar^{2}$, where $n(x)$ is the spherical end-toend distribution, i.e. the Fourier transform of $n(p)$, while the second term is the free particle contribution which is independent of the environment [23]. $f(x)$ was derived from the experimental detector-averaged $\bar{J}(y)$ data obtained after correcting the detectoraveraged NCP data (simple average without error weighting) $\bar{F}(y)$, for Final State Effects $\Delta J(y, q)$. The corresponding expression is [20]

$f(x)=-\frac{M x}{\beta \hbar^{2}}+\frac{\int_{0}^{\infty} d y y \sin (x y / \hbar) \bar{J}(y)}{\hbar \int_{0}^{\infty} d y \cos (x y / \hbar) \bar{J}(y)}$

This analysis was used in Ref. [20] to complement the standard nonlinear fitting analysis, Eq. (1.9), providing further confirmation that indicating that the quantum state of the proton in ice is well represented by a quasi-harmonic anisotropic motion.

In this work we propose and assess the direct determination of the momentum distribution from the application of Eq. (1.3) to the experimental Compton profile, $\bar{F}(y)$, obtaining

$n^{*}(p)=-\left.\frac{1}{2 \pi y} \frac{d \bar{F}(y)}{d y}\right|_{y=p}$

i.e. neglecting the effect of resolution and FSE broadening. The use of Eq. (1.11) can be introduced by considering the properties of the derivative of a convolution [24]:

$\frac{d F_{l}(y, q)}{d y}=\frac{d\left[J(y)+\Delta J_{l}(y, q)\right]}{d y} \otimes R_{l}(y, q)$

The subsequent application of the distribution properties of the convolution then allows us to extend the derivative to $\bar{F}(y)$ :

$\frac{d \bar{F}(y)}{d y}=\frac{d[\bar{J}(y)+\overline{\Delta J(y)}]}{d y} \otimes \bar{R}(y)$

\section{Proof of concept and application to experimental neutron Compton profiles}

The differences between the "true" $n^{*}(p)$ and the "true" $n(p)$ are the resolution broadening and the derivative of the (resolution-broadened) FSE term. Recent developments on detector technologies and energy selection methods have allowed us to optimise and reduce the resolution broadening to approximately 13\% FWHM of the Compton profile, for proton momentum distribution studies [10,25-27]. The FSE are of the order of $1 / q$ or smaller, therefore the modulation due to FSE in the signal is of the order of less than $3 \%$ for the typical wave vectors values accessed on VESUVIO [9]. In order to assess the extent to which $n^{*}(p)$ can be considered as a reliable determination of $n(p)$, we have first carried out a proof of concept, analysing the data to extract $n^{*}(p)$ from a DINS Monte Carlo simulation of known values of $n(p)$, FSE, and resolution. We then apply the method to a real experiment, extracting the proton's $n^{*}(p)$ for a polycrystalline ice Ih at $271 \mathrm{~K}$, whose analysis in terms of parametric fitting was presented in Ref. [20], and compare the two determinations.

The simulation consists of a set of Monte Carlo simulated experimental Compton profiles for a proton-containing system. The DINSMS code [28] incorporates the resolution in a virtually exact manner, tracking and weighting individual neutron histories by probability distribution function for the geometrical uncertainties, emission time from the moderator, energy analyser transfer function, and other parameters. Applying the method to such synthetic data is a self-consistent test providing a proof of concept. The sample was monoatomic hydrogen with isotropic Gaussian momentum distribution with standard deviation $\sigma_{M C}=4.70 \AA^{-1}$. Final state effects were also included assuming an isotropic harmonic proton effective potential with mean 


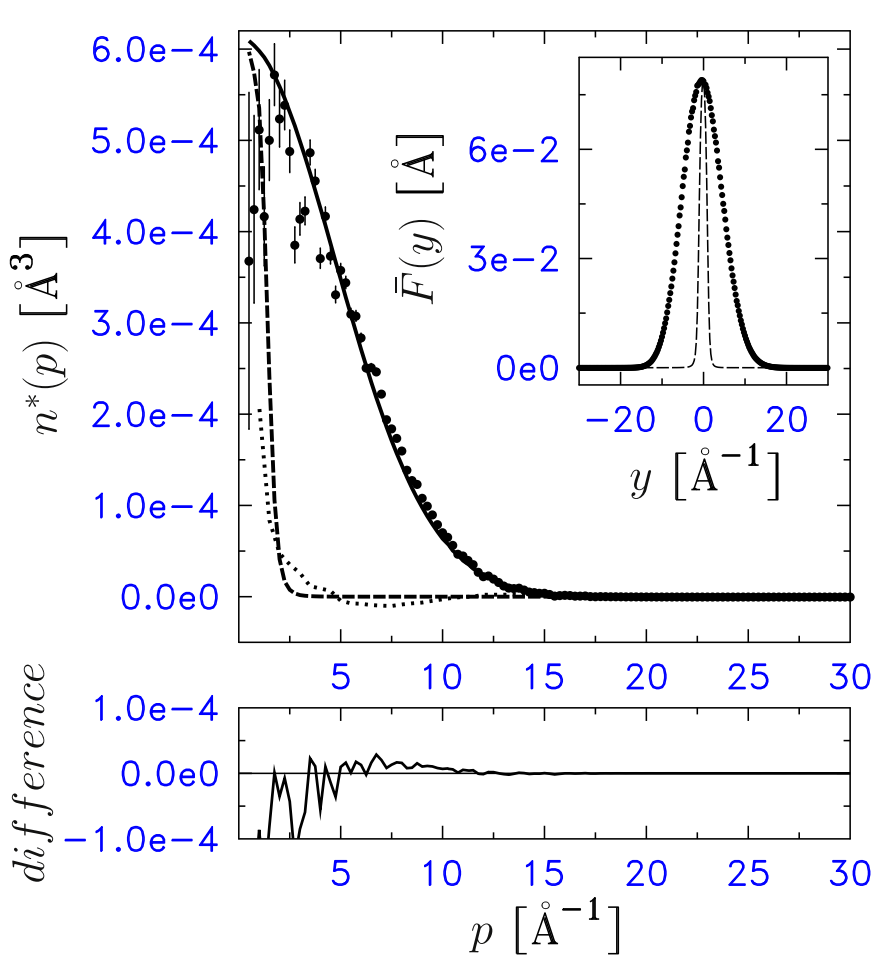

Fig. 1. Direct numerical determination of the momentum distribution, $n^{*}(p)$ from the DINSMS Monte Carlo simulation (points with error bars); input momentum distribution of the simulation, $n(p)$, representing the target momentum distribution to be reconstructed, continuous line; resolution in momentum space, dashes line; contribution arising from the FSE terms, dotted line. The lower panel reports the difference between $n^{*}(p)$ and the "true" $n(p)$. The inset reports $\bar{F}(y)$ (points with error bars) and $\bar{R}(y)$ (dashed line).

frequency $\hbar \bar{\omega}=275 \mathrm{meV}$, including terms up to $1 / q^{2}$ (see Eq. (1.7)). Standard data reduction [5] was carried out on these data to obtain $F_{l}(y, q), R_{l}(y, q)$ and $\bar{F}(y), \bar{R}(y)$. Eq. (1.11) was then applied to $\bar{F}(y)$ using a three-point numerical derivative and the results are reported in Fig. 1. From this figure it appears that the low momentum region of $n^{*}(p)$ shows scattered intensities, up to approximately $3 \AA^{-1}$. This feature is due to the presence of FSE in the simulation, which introduce a slight asymmetry in $\bar{F}(y)$, causing an effective shift to negative $y$, that is amplified when the derivative is calculated near the origin, $n^{*}(p \simeq 0)=-\left.(1 / 2 \pi y)(d \bar{F}(y) / d y)\right|_{y \simeq 0}$. This is however reassuring that the simulation captures this feature, typically present in the experimental spectra. On the other hand, data above $3 \AA^{-1}$ are considerably smooth and provide an accurate detail of the line shape. Moreover, the agreement between $n^{*}(p)$ and $n(p)$ is remarkable, as shown by their difference, which is always below $0.2 \times 10^{-4}$. This behaviour reflects the shape of the FSE component, decaying rapidly above $5 \AA^{-1}$ (about one standard deviation). The resolution component is again very narrow and shows a rapid decay above $2 \AA^{-1}$. These findings imply that $n^{*}(p)$ is an accurate representation of the "true" momentum distribution at intermediate and high momenta, where the non-Gaussian (anharmonic and/or anisotropic) character of the momentum distribution in molecular and quantum systems is more prominent $[5,23]$.

To test these hypotheses on more realistic grounds, we have carried out an analogous derivation of $n^{*}(p)$ for the water's proton in polycrystalline ice at $271 \mathrm{~K}$, from the DINS experiment of Flammini et al. [20]. The momentum distribution, $n(p)$, for this sample has been derived using parametric fitting methods, as well as numerical evaluation of the variance and of the proton mean force. Similar to the previous case, a three point numerical derivative was carried out for $\bar{F}(y)$ and the results are reported in Fig. 2.

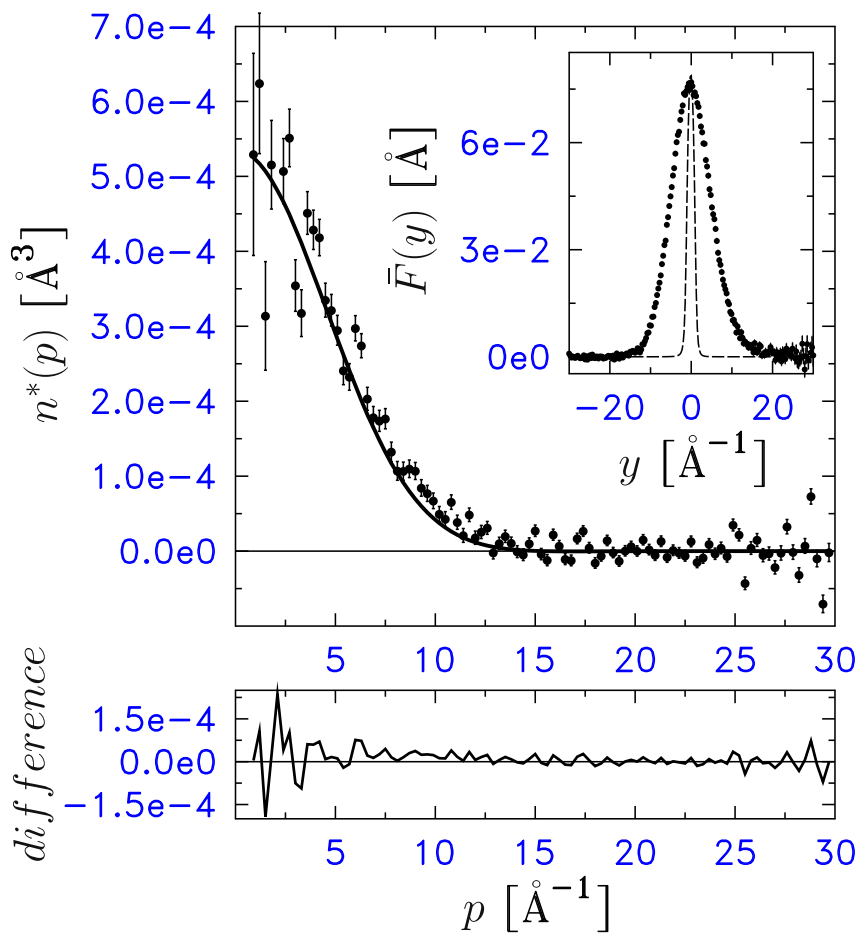

Fig. 2. Direct numerical determination of the proton momentum distribution, $n^{*}(p)$, for polycrystalline ice at $271 \mathrm{~K}$ (points with error bars); momentum distribution derived by Flammini et al. [20], $n(p)$, continuous line. The lower panel reports the difference between $n^{*}(p)$ and $n(p)$. The inset reports $\bar{F}(y)$ (points with error bars) and $\bar{R}(y)$ (dashed line).

The method, applied to an experimental data set, shows the principal features already reported in the simulation that was used as a proof of concept: the larger noise present at low momenta, and subsequent substantial agreement between $n^{*}(p)$ and $n(p)$ above $3 \AA^{-1}$. This demonstrates that $n^{*}(p)$ can provide a determination of the underlying momentum distribution to a good degree, without resorting to multiparametric fitting. Theoretical models of momentum distributions can be then directly compared to $n^{*}(p)$, without going through the intermediate steps of fitting. The quantitative estimates of the degree of accuracy required for the optimised determination of $n^{*}(p)$ are out of the scope for the present study, but can be enumerated as follows: (1) optimisation of the numerical derivative with more refined techniques (five point derivatives, filtering, etc.), in order to reduce the noise; (2) optimised weighting of the contributions arising from positive and negative $y$, which have intrinsically different noise and uncertainties [20]; and (3) subtraction of the FSE-dependent modulation before or after the determination of $n^{*}(p)$. In particular, these would improve the determination of the standard deviation, $\sigma$, of the momentum distribution, which is directly related to the proton zero-point mean kinetic energy. In the present case, numerical integration of the second moment of the radial momentum distributions obtained from $n^{*}(p)$ gives the following results: (1) for the simulated data $\sigma^{*}=4.7 \pm 0.2 \AA^{-1}$, to be compared with the input value $\sigma_{M C}=4.70 \AA^{-1} ;(2)$ for the experimental data $\sigma^{*}=5.1 \pm 1.4 \AA^{-1}$, to be compared with the values obtained from the line shape analysis in Ref. [20], $\sigma=4.99 \pm 0.03 \AA^{-1}$, and from the numerical integration of Compton profiles, $\sigma=5.2 \pm 0.3 \AA^{-1}$ [20]. It therefore appears that, at present, the best non-parametric determination of the kinetic energies can be carried out using the integration of neutron Compton profiles [21], while the determination of $n^{*}(p)$ allows the non-parametric determination of the line shapes of the momentum distributions. 


\section{Discussion and conclusions}

The work presented here is part of a series of approaches for data analysis and interpretation proposed recently to enhance and strengthen the scope of $\mathrm{eV}$ neutron scattering for the determination of atomic momentum distributions [20-23,29]. The direct numerical determination of the momentum distribution can be used to unambiguously identify nuclear quantum effects, the chemical environment, and the anisotropic-harmonic or anharmonic character of atomic motions in condensed systems. Powerful theoretical schemes are currently underway to provide quantitative comparison with first-principles atomistic modeling [30,31], and efforts for the advances in analysis and interpretation of experiments play a fundamental role for both reliable testing of atomistic modeling, and more importantly, to provide experimental results that stimulate further theoretical investigations. The method proposed here is intended to be part of a set of non-parametric determinations of the momentum distributions [20-23] that can be used synergistically with the parametric fitting analysis, broadening the capabilities of the deep inelastic neutron scattering technique. Moreover, the direct numerical determination of $n^{*}(p)$ can be applied to the recent studies of heavier mass systems, such as lithium and oxygen [31,32], that aim at assessing the magnitude of nuclear quantum fluctuations in any condensed matter system.

\section{Acknowledgements}

This work was supported within the CNR-STFC Agreement No. $01 / 9001$ concerning collaboration in scientific research at the spallation neutron source ISIS. The financial support of the Consiglio Nazionale delle Ricerche in this research is hereby acknowledged.

\section{References}

[1] G.E. Jauncey, Physical Review 25 (1925) 314

[2] J.W. Du Mond, Physical Review 33 (1929) 643.

[3] V.F. Sears, Physical Review B 30 (1984) 44

[4] A. Pietropaolo, R. Senesi, Physics Reports 508 (2011) 45.

[5] C. Andreani, D. Colognesi, J. Mayers, G.F. Reiter, R. Senesi, Advances in Physics 54 (2005) 377.
[6] G.I. Watson, Journal of Physics: Condensed Matter 8 (1996) 5955.

[7] S.W. Lovesey, The Theory of Neutron Scattering from Condensed Matter, Oxford University Press, Oxford, 1986.

[8] G.B. West, Physics Reports 18 (1975) 263.

[9] J. Mayers, G. Reiter, Measurement Science and Technology 23 (2012) 045902.

[10] S. Imberti, C. Andreani, V. Garbuio, G. Gorini, A. Pietropaolo, R. Senesi, M. Tardocchi, Nuclear Instruments and Methods in Physics Research Section A 522 (2005) 463.

[11] R.C. Blasdell, R.O. Simmons, Nuclear Instruments and Methods in Physics Research Section A 405 (1998) 71.

[12] D.M. Ceperley, R.O. Simmons, R.C. Blasdell, Physical Review Letters 77 (1996) 115.

[13] S.O. Diallo, R.T. Azuah, O. Kirichek, J.W. Taylor, H.R. Glyde, Physical Review B 80 (2009) 060504

[14] S.O. Diallo, J.V. Pearce, R.T. Azuah, O. Kirichek, J.W. Taylor, H.R. Glyde, Physical Review Letters 98 (2007) 205301.

[15] S.O. Diallo, J.V. Pearce, R.T. Azuah, F. Albergamo, H.R. Glyde, Physical Review B 74 (2006) 144503

[16] D.G. Narehood, M.K. Kostov, P.C. Eklund, M.W. Cole, P.E. Sokol, Physical Review B 65 (2002) 233401.

[17] K.W. Herwig, P.E. Sokol, T.R. Sosnick, W.M. Snow, R.C. Blasdell, Physical Review B 41 (1990) 103.

[18] D. Homouz, G. Reiter, J. Eckert, J. Mayers, R. Blinc, Physical Review Letters 98 (2007) 115502.

[19] C. Pantalei, A. Pietropaolo, R. Senesi, S. Imberti, C. Andreani, J. Mayers, C. Burnham, G. Reiter, Physical Review Letters 100 (2008) 177801

[20] D. Flammini, A. Pietropaolo, R. Senesi, C. Andreani, F. McBride, A. Hodgson, M.A. Adams, L. Lin, R. Car, Journal of Chemical Physics 136 (2012) 024504.

[21] R. Senesi, Nuclear Instruments and Methods in Physics Research A 661 (2012) 70.

[22] M. Krzystyniak, M.A. Adams, A. Lovell, N.T. Skipper, S.M. Bennington, J. Mayers, F. Fernandez-Alonso, Faraday Discussions 151 (2011) 171.

[23] L. Lin, J.A. Morrone, R. Car, M. Parrinello, Physical Review Letters 105 (2010) 110602.

[24] R. Bracewell, The Fourier Transform and Its Applications, 3 ed. McGraw-Hill Science/Engineering/Math, ADDRESS, 1999.

[25] E.M. Schooneveld, J. Mayers, N.J. Rhodes, A. Pietropaolo, C. Andreani, R. Senesi, G. Gorini, E. Perelli-Cippo, M. Tardocchi, Review of Scientific Instruments 77 (2006) 5103.

[26] A. Pietropaolo, C. Andreani, A. Filabozzi, R. Senesi, G. Gorini, E. Perelli-Cippo, M. Tardocchi, N.J. Rhodes, E.M. Schooneveld, Journal of Instrumentation 1 (2006) P04001.

[27] A. Pietropaolo, G. Gorini, G. Festa, C. Andreani, M.P. de Pascale, E. Reali, F. Grazzi, E.M. Schooneveld, Review of Scientific Instruments 80 (2009) 095108.

[28] J. Mayers, A.L. Fielding, R. Senesi, Nuclear Instruments and Methods in Physics Research Section A 481 (2002) 454

[29] R. Senesi, A. Pietropaolo, C. Andreani, Nuclear Instruments and Methods in Physics Research A 594 (2008) 244.

[30] M. Ceriotti, D.E. Manolopoulos, Physical Review Letters 109 (2012) 100604.

[31] A.G. Seel, M. Ceriotti, P.P. Edwards, J. Mayers, Journal of Physics: Condensed Matter 24 (2012) J5401.

[32] A.G. Seel, A. Sartbaeva, J. Mayers, A.J. Ramirez-Cuesta, P.P. Edwards, Journal of Chemical Physics 134 (2011) 114511. 